\title{
RETOS PARA LA INCLUSIÓN LABORAL DE PERSONAS CON DISCAPACIDAD EN COSTA RICA
}

\section{CHALLENGES FOR THE LABOR INCLUSION OF PERSONS WITH DISABILITIES IN COSTA RICA}

\section{DESAFIOS PARA A INCLUSÃO LABORAL DE PESSOAS COM DEFICIÊNCIA NA COSTA RICA}

\section{Héctor Andrés Canossa Montes de Oca ${ }^{1}$}

\begin{abstract}
Resumen
Más del $18 \%$ de los costarricenses mayores de 18 años son personas con discapacidad ( $\mathrm{PcD})$, además la desocupación laboral en este grupo es mayor que la de la población general, alcanzando el $60 \%$. Este artículo busca motivar la igualdad de oportunidades de empleo, por lo que es importante analizar las implicaciones de la inclusión laboral, identificando las repercusiones en las empresas y en los empleados con discapacidades. Para este propósito, se realizó una investigación descriptiva, a través de una revisión literaria y aplicando encuestas y entrevistas, revelando la poca experiencia de las compañías que contrataron a $\mathrm{PCD}$ y el desconocimiento de sus beneficios; además, la percepción general de que no existe una promoción efectiva de la inclusión por parte del gobierno costarricense. Las empresas consideran como desafíos principales la falta de perfiles de puestos inclusivos y la inversión en adaptaciones físicas, mientras que las $\mathrm{PcD}$ consideran que los desafíos residen en capacitación, cambios en procesos de reclutamiento y selección, los perfiles de puesto y principalmente la actitud de los empleadores.
\end{abstract}

Palabras clave: empresa inclusiva, estrategia, talento humano, discapacidad, igualdad.

Doi: https://doi.org/10.15359/eys.25-58.4

Recibido: 31-07-2020. Reenvíos: 20-10-2020, 23-10-2020, 26-10-2020. Aceptado: 02-11-2020. Publicado: 04-12-2020.

1 MBA. Académico de la Universidad Nacional en la Sede Regional Chorotega, la Universidad Técnica Nacional y Maestría en Administración de Empresas del Tecnológico de Costa Rica. Correo electrónico: hector.canossa.montesdeoca@una.cr. ORCID: https://orcid.org/0000-0003-0952-3193. 


\begin{abstract}
More than $18 \%$ of Costa Ricans over the age of 18 are persons with disabilities (PwD). Reaching 60\%, unemployment in this group is greater than the rate in the general population. This article aims to motivate equal employment opportunities, for which it is important to analyze the implications of labor inclusion, by identifying its repercussions on companies and employees with disabilities. For this purpose, a descriptive research was conducted including a review of literature, as well as surveys and interviews. The companies hiring PwDs showed little experience and lack of knowledge regarding their benefits, In addition, there is a general perception that there is no effective promotion of inclusion by the Costa Rican government. Companies consider the lack of adequate job profiles and investment in physical adaptations as their main challenges, while PwDs consider lack of training, changes in recruitment and selection processes, job profiles, and mainly the employers' attitude as their main challenges.
\end{abstract}

Keywords: inclusive organization, strategy, human talent, disability, equality.

\title{
Resumo
}

Mais de $18 \%$ dos costarriquenhos com mais de 18 anos são pessoas portadora de deficiência (PPD), e o desemprego nesse grupo é maior do que a da população em geral, chegando a $60 \%$. Este artigo busca motivar a igualdade de oportunidades de emprego, por isso é importante analisar as implicações da inclusão laboral, identificando o impacto sobre as empresas e os funcionários com deficiência. Para tanto, foi realizada uma pesquisa descritiva por meio de revisão bibliográfica e aplicação de pesquisas e entrevistas, revelando a pouca experiência das empresas que contrataram PPD e o desconhecimento de seus benefícios; além disso, a percepção geral de que não há uma divulgação efetiva da inclusão por parte do governo costarriquenho. As empresas consideram como principais desafios a falta de perfis de posição inclusiva e o investimento em acomodações físicas, enquanto os PDDs consideram que os desafios sejam a formação, as mudanças nos processos de recrutamento e seleção, os perfis profissionais e, principalmente, a atitude dos empregadores.

Palavras-chave: empresa inclusiva, estratégia, talento humano, deficiência, igualdade.

\section{Introducción}

Para iniciar, surge una interrogante que expone el problema de investigación: ¿Cuáles han sido las implicaciones de la inclusión laboral, tanto para las empresas costarricenses como para los colaboradores con discapacidad? 
La definición de discapacidad, según el artículo 2 de la Ley 7600 de la Asamblea Legislativa de Costa Rica (1996), es "cualquier deficiencia física, mental o sensorial que limite, sustancialmente, una o más de las actividades principales de un individuo".

En el país, mediante leyes se ha buscado fomentar la igualdad en oportunidades para PcD, pero además de las leyes es necesario el aporte de la sociedad para ser realmente inclusiva; en esta investigación se aborda el tema desde la perspectiva empleo y sus implicaciones, donde se ve la discapacidad como la combinación de la limitación con las barreras que existen en el entorno. Siguiendo el modelo social, como expone Victoria (2013) ya no se visualiza este tema desde un modelo rehabilitador sino como un modelo social, que significa entender la discapacidad como una cuestión de derechos humanos, valorando más las capacidades del humano, el entorno inmediato donde la familia es relevante y el medio como portador de oportunidades equitativas y eliminación de barreras (p. 819-820).

Estas iniciativas tanto en Costa Rica como en Latinoamérica ha incentivado una visibilidad mayor en los últimos años, esto entre otros aspectos se encuentra relacionado con la Convención sobre los Derechos de las PcD de las Naciones Unidas del año 2006 (González y Stang, 2014, p.69), pero de ahí mismo surge el cuestionamiento entre las iniciativas y las acciones tangibles.

Es importante reconocer la relevancia del tema para el país y a través de datos estadísticos se conoce que el 18,2\% de los costarricenses mayores de 18 años tiene una situación de discapacidad, como se muestra en la tabla 1, además hay más mujeres con discapacidad que hombres, y la mayor parte de estas personas se encuentran en edades entre 36 y 64 años, cabe destacar que alrededor de la mitad se encuentran en unión libre o casados.

\section{Tabla 1}

Datos generales de personas mayores de 18 años por condición de discapacidad, 2018

\begin{tabular}{lcccccc}
\hline & \multicolumn{2}{c}{ Total de personas } & \multicolumn{2}{c}{$\begin{array}{c}\text { Personas sin situación } \\
\text { de discapacidad }\end{array}$} & $\begin{array}{c}\text { Personas con } \\
\text { Discapacidad }\end{array}$ \\
\hline & Absolutos & Relativos & Absolutos & Relativos & Absolutos & Relativos \\
$\begin{array}{l}\text { Total costarricenses } \\
\text { mayores de 18 años }\end{array}$ & 3.677 .722 & $100 \%$ & 3.007 .082 & $81,8 \%$ & 670.640 & $18,2 \%$ \\
Sexo & & & & & & \\
Hombres & 1.843 .472 & $100 \%$ & 1.581 .521 & $85,8 \%$ & 261.951 & $14,2 \%$ \\
$\begin{array}{l}\text { Mujeres } \\
\text { Grupos de edad }\end{array}$ & 1.834 .251 & $100 \%$ & 1.425 .561 & $77,7 \%$ & 408.689 & $22,3 \%$ \\
$\begin{array}{l}\text { De 18 a 35 años } \\
\text { De 36 a 64 años }\end{array}$ & 1.256 .440 & $100 \%$ & 1.135 .930 & $90,4 \%$ & 120.510 & $9,6 \%$ \\
$\begin{array}{l}\text { De 65 años y más } \\
\text { Estado conyugal }\end{array}$ & 617.803 .389 & $100 \%$ & 1.470 .143 & $81,5 \%$ & 333.246 & $18,5 \%$ \\
En unión libre o & $100 \%$ & 401.009 & $64,9 \%$ & 216.884 & $35,1 \%$ \\
casados(as) & 2.039 .171 & $100 \%$ & 1.698 .363 & $83,3 \%$ & 340.808 & $16,7 \%$
\end{tabular}




\begin{tabular}{lcccccc}
\hline & Total de personas & \multicolumn{2}{c}{$\begin{array}{c}\text { Personas sin situación } \\
\text { de discapacidad }\end{array}$} & $\begin{array}{c}\text { Personas con } \\
\text { Discapacidad }\end{array}$ \\
\hline $\begin{array}{l}\text { Divorciados(as), } \\
\text { separados(as) } 0\end{array}$ & 719.076 & $100 \%$ & 524.309 & $72,9 \%$ & 194.767 & $27,1 \%$ \\
$\begin{array}{l}\text { viudos(as) } \\
\text { Solteros(as) }\end{array}$ & 919.475 & $100 \%$ & 784.410 & $85,3 \%$ & 135.065 & $14,7 \%$ \\
\hline
\end{tabular}

Fuente: Elaboración propia sobre la base de la Encuesta Nacional sobre Discapacidad 2018 del Instituto Nacional de Estadísticas y Censos y el Consejo Nacional de PcD de Costa Rica (2019).

Al considerar dos aspectos puntuales que son relevantes para valorar las razones de la desocupación laboral, como son el grado de discapacidad y la posición en el empleo, cabe destacar como se muestra en el tabla 2, que el 79\% tiene una discapacidad severa, esto quiere decir que son personas que presentaron una dificultad mayor o más intensa al realizar actividades cotidianas, esto es relevante ya que evidentemente al tener una situación severa el acceso al empleo enfrentará mayores retos (INEC y CONAPDIS, 2019).

Tabla 2

Datos generales de PCD mayores de 18 años por grado de discapacidad y posición en el empleo, 2018

\begin{tabular}{lll}
\hline & Absolutos & Relativos \\
\hline $\begin{array}{l}\text { Total de PcD } \\
\text { Grado de discapacidad }\end{array}$ & 670.640 & $100,0 \%$ \\
Personas en situación de discapacidad severa & & \\
Personas en situación de discapacidad de leve a moderada & 1331.605 & $79,3 \%$ \\
Posición en el empleo & & $20,7 \%$ \\
Fuera de la fuerza de trabajo & 378.013 & $56,4 \%$ \\
Ocupados(as) & 267.167 & $39,8 \%$ \\
Desocupados(as) & 25.460 & $3,8 \%$ \\
\hline
\end{tabular}

Nota: personas que presentan discapacidad de leve a moderada tienen dificultad menor al realizar actividades cotidianas; severa son personas que presentaron dificultad mayor al realizar actividades cotidianas.

Fuente: Elaboración propia sobre la base de la Encuesta Nacional sobre Discapacidad 2018 del INEC y CONAPDIS (2019).

Considerando la tabla 2, se identifica que del total de PcD, 43,6\% forman parte de la población económicamente activa (PEA) es decir 292.627 personas, es decir, los que no están fuera de la fuerza de trabajo y de estas 25.460 están desocupadas, por consiguiente $8.7 \%$ de desempleo.

Comparando las PcD con las que no presentan una situación de discapacidad, porcentualmente hay una brecha evidente mostrada en la figura 1 , donde los ocupados mayores de 18 años que no presentan discapacidad alcanzan el 64\%, y por contraparte 
las PcD llegan hasta $40 \%$, y en el punto de fuera de la fuerza de trabajo ocurre algo contrario, debido como se mencionó anteriormente por el grado de discapacidad.

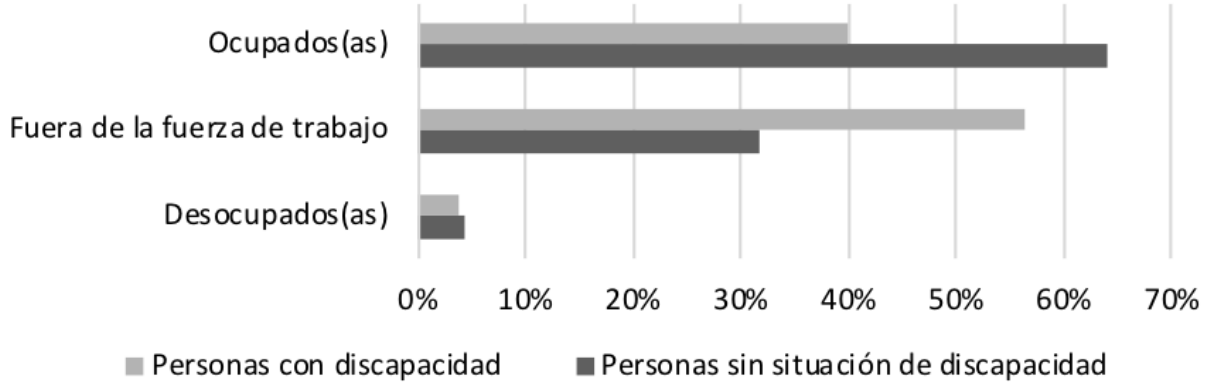

Figura 1. Costa Rica (posición en el empleo): comparación de personas mayores de 18 años sin situación de discapacidad y PcD (en porcentajes).

Fuente: Elaboración propia con base en Encuesta Nacional sobre Discapacidad 2018 del INEC (2019a).

En relación con la figura anterior cabe agregar que de las 670.640 personas que tienen una situación de discapacidad, 311.214 personas reciben asistencia personal, es decir el $46,4 \%$ lo que reducirá las posibilidades de que estas quieran ser incluidas en el contexto laboral. Este apoyo personal es el apoyo humano que se brinda hacia una PcD para realizar las actividades cotidianas y tener autonomía, estas incluyen actividades como caminar, alimentarse, salir a la calle, comunicarse, la realización trabajo doméstico, autocuidado, entre otros (INEC y CONAPDIS, 2019).

Esta investigación se justifica al considerar relevante la responsabilidad empresarial de promover una igualdad de oportunidades en los procesos del área, tales como reclutamiento, selección y contratación. El trabajo se elabora para dar a conocer la posición empresarial ante la contratación de las $\mathrm{PCD}$, sus retos y beneficios. Además, de aportar socialmente a la inclusión laboral, en la que Costa Rica ha ratificado la Convención sobre los Derechos de las Personas con Discapacidad (Ley 8661, Decreto Ejecutivo $\mathrm{N}^{\circ} 34780$ de 29 de setiembre de 2008).

Parte clave del éxito de una organización, como expresan Mathis, Jackson y Valentine (2014) es "el capital humano que no son solo las personas en las organizaciones, sino que también lo que esas personas contribuyen al éxito (...) es el valor colectivo de las capacidades, conocimientos, habilidades, experiencias de vida, y la motivación de una fuerza de trabajo" (p.6).

Por consiguiente, las organizaciones deben promover igualdad de oportunidades para tener un capital humano competitivo, y más que limitar una contratación por un tema de discapacidad física, mental o sensorial, debe tener un enfoque en las capacidades de la persona y el valor que puede agregar a la organización. 
El objetivo general de este artículo es analizar las implicaciones de la inclusión laboral, tanto para las empresas costarricenses como para los colaboradores con discapacidad, además específicamente se pretende conocer las implicaciones de la inclusión laboral para las empresas costarricenses, demostrar las implicaciones de la inclusión laboral en los colaboradores con discapacidad en dichas empresas y realizar recomendaciones que permitan, cada vez más, una mayor inclusión laboral de las PcD.

Estos objetivos se alcanzan mediante una investigación que involucra revisión literaria, encuestas y comunicaciones personales que agregan valor mediante diversos puntos de vista. Con esta investigación se pretende informar a empresas y $\mathrm{PcD}$, sobre beneficios y retos, buscando promover la inclusión laboral. Además, el artículo 23 de la Ley $N^{\circ} 7600$ (1996) señala que el Estado garantizará a las PCD, tanto en zonas rurales como urbanas, el derecho de un empleo adecuado a sus condiciones y necesidades personales. Esto, sin duda, es un reto ya que no solo depende de legislación sino, también, de las empresas.

\section{Revisión literaria}

Desde la disciplina de las ciencias empresariales es fundamental promover iniciativas hacía decisiones más inclusivas y la promoción del capital humano dentro de las organizaciones, en ese sentido, la revisión de conceptos, legislación e iniciativas orientan la discusión. La forma en que una PcD puede conseguir un empleo, va más allá de la promoción por parte del Estado, pues se debe considerar la aceptación de las empresas y mayor apertura a este mercado laboral, así también que la PcD desarrolle actitudes y aptitudes necesarias para ocupar un puesto.

Sobre el concepto de inclusión laboral de PcD, incluye el hecho de facilitar a este grupo el espacio donde puedan aprender a trabajar en la medida de sus posibilidades, darles respeto como seres humanos con capacidades, es brindarles la oportunidad de demostrar sus potencialidades, acompañándolos para que desarrollen sus capacidades de producción y de brindar servicio eficiente y responsable (Peressotti, 2017, p.18).

Como contraparte, la exclusión laboral persiste en un mundo que, de manera contradictoria, aparenta ser cada vez más abierto, inclusivo y progresivo. La exclusión laboral sin lugar a duda es un problema que afecta directamente de forma más intensa a la población más vulnerable (Millán, Arredondo y Vázquez, 2018, p.175).

Según la Organización Internacional del Trabajo (2015) los índices de desempleo de las PcD son elevados y los índices de participación en el mercado laboral son muy inferiores a los de las personas sin discapacidad, entonces, la inclusión de la discapacidad significa promover y garantizar la participación de las PcD en la educación, la formación y el empleo y en todos los aspectos de la sociedad, así como brindar los apoyos necesarios y ajustes razonables a dichas personas para permitir su plena participación.

Sobre elementos que obstruyen la inclusión cabe mencionar el menor nivel formativo disonante con las demandas de las empresas, la permanencia de estereotipos y prejuicios sociales vinculados al potencial laboral de la población con discapacidad, los déficits 
de accesibilidad de los espacios físicos y la subsistencia de políticas pasivas (Mercado, Aizpurúa y García, 2013, p.99).

La educación en las PCD es importante para la obtención de empleo, en Costa Rica hay una brecha en los niveles de instrucción, en la figura 2 se muestra que en los niveles superiores es más notable la brecha: 17,1\% cuentan con educación superior mientras que de las personas sin situación de discapacidad alcanza el $24,7 \%$.

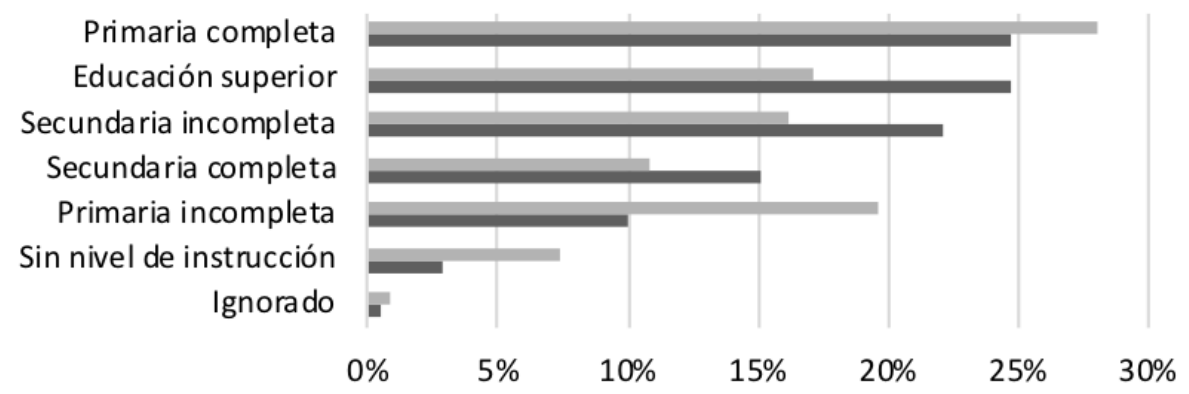

- Personas con discapacidad

- Personas sin situación de discapacidad

Figura 2. Costa Rica (niveles de instrucción): comparación de personas sin situación de discapacidad y PCD (en porcentajes).

Fuente: Elaboración propia sobre la base de la Encuesta Nacional sobre Discapacidad 2018 del Instituto Nacional de Estadísticas y Censos (2019b).

En Costa Rica, el Ministerio de Educación Pública (MEP) ha mostrado esfuerzos para reducir está brecha a través del Centro de Educación Especial donde pueden ingresar personas desde 0 hasta 21 años, cuentan con 22 centros de educación especial alrededor del país (Ministerio de Educación Pública de Costa Rica, 2019).

Entonces se presenta la necesidad de un doble enfoque, una parte en iniciativas específicas destinadas a superar desventajas o barreras particulares y, como segundo enfoque, garantizar la inclusión en los servicios y actividades de carácter general, tales como la formación profesional y el empleo. La legislación que aporta a la inclusión laboral en Costa Rica incluye todo marco normativo donde se involucra la discapacidad y el empleo.

Desde el 2012 Costa Rica cuenta con el Plan Nacional de Inserción Laboral para PcD, lo cual permite visualizar un interés por mejorar la situación actual. Como ejemplo, la realización de talleres con participación de representantes de 140 instituciones públicas; en este caso, la Presidencia de la República de Costa Rica (2017a) indicó: "busca lograr mayor orientación para la aplicación efectiva de la Ley 8862, Inclusión y protección laboral de las PCD en el sector público". La ley en cuestión señala que en las ofertas de 
empleo público de los poderes del Estado se reservará cuando menos un porcentaje de un $5 \%$ de las vacantes (Ley 8862, 2010).

Según el INEC (2011) en el Censo 2011 de Costa Rica considerandos no solo mayores de 18 años, el porcentaje de PcD es de 10,53\%, y cabe agregar que la discapacidad que se presenta con mayor frecuencia es la visual, con 55,53\%. Además, la limitación para caminar o subir gradas alcanza el $31 \%$ de las PcD; este es un aspecto por considerar ya que puede influir en las contrataciones por los tipos de puestos y adecuaciones requeridas.

Además, en capacitación para PcD, surgen oportunidades en áreas de demanda laboral, mediante Empléate Inclusivo. Barquero (2016) indica que el objetivo de este programa del MTSS, en relación con las PCD, es mejorar la empleabilidad para potenciar la inserción laboral de PcD en puestos de calidad.

Considerando el impacto de las leyes en la inclusión laboral de las PcD, cabe destacar las leyes 9303, 7600 y 7092. Con la Ley 9303 (2015) se crea el Consejo Nacional de PCD (Conapdis), rector en discapacidad, adscrito al Ministerio de Trabajo y Seguridad Social (MTSS). Entre sus funciones, según artículo 3 inciso $f$ es "promover y velar por la inclusión laboral de persona con discapacidad en los sectores público y privado, en coordinación con el MTSS y otros servicios de intermediación de empleo, así como velar por su cumplimiento".

En la Ley 7600 (1996), artículo 3 sobre objetivos, inciso b, se indica que uno de estos objetivos es garantizar la igualdad de oportunidades para la población y, entre esas, la educación y empleo; además, en el artículo 2 se expone el concepto de equiparación de oportunidades: "proceso de ajuste del entorno, los servicios, las actividades, la información, la documentación, así como las actitudes a las necesidades de las personas, en particular de las discapacitadas".

Por su parte, el Ministerio de Trabajo y Seguridad Social tiene el Departamento de Igualdad de Oportunidades para las PcD, encargado de divulgar la legislación vigente en materia de discapacidad, y cabe destacar que dos de sus funciones son asesorar al sector empresarial en materia de equiparación de oportunidades para PcD y promover la responsabilidad social empresarial como una acción afirmativa en la contratación de PcD, a fin de mejorar las condiciones de vida de estas personas y sus familias (MTSS, 2019).

Considerando a los empleadores, la Ley 7092 (1988), sobre el impuesto a la renta, muestra un incentivo en favor de los que contraten PcD; en el artículo 8, inciso b, se indica que son deducibles a la renta bruta las formas de compensación, adecuaciones al puesto y adaptaciones al sitio e indica que, además, podrá deducirse una cantidad igual adicional a la que se pague por los conceptos de compensación.

Por otra parte, destaca el premio Costa Rica Incluye que reconoce a empresas por buenas prácticas de inclusión social y laboral de PcD, impulsado por el Ministerio de Trabajo y Seguridad Social (MTSS), la Asociación Empresarial para el Desarrollo (AED), la Segunda Vicepresidencia de la República, el Consejo Nacional de las PcD (Conapdis), en 
alianza con el Programa de Naciones Unidas para el Desarrollo (PNUD) y la Organización Internacional del Trabajo (OIT) (Presidencia de la República de Costa Rica, 2017b).

La Declaración Universal de Derechos Humanos (ONU, 1948), en el artículo 1, expone: "Todos los seres humanos nacen libres e iguales en dignidad y derechos y, dotados como están de razón y conciencia, deben comportarse fraternalmente los unos con los otros". Además, parte del artículo 23 indica: "Toda persona tiene derecho al trabajo, a la libre elección de su trabajo, a condiciones equitativas y satisfactorias de trabajo y a la protección contra el desempleo".

En Costa Rica, en el artículo 4 de la Ley 8661 (2008) que aprueba la Convención sobre los Derechos de las PcD, se indica que en el país se deben tomar todas las medidas pertinentes para que ninguna persona, organización o empresa privada discrimine por motivos de discapacidad.

Esto demuestra la relevancia por hacer realidad la inclusión laboral, pero ¿qué es una empresa inclusiva?; según la Red de Empresas Inclusivas Costa Rica (2013) es

aquella que valora las diferencias, cree en diversidad y comprende cómo esta afecta cada parte de su negocio y asume el desafío de mejorar las relaciones con su entorno, aumentar su productividad y construir una mayor cercanía con todas sus partes interesadas. Una empresa inclusiva ha trabajado para que sus procesos de reclutamiento y selección no sean discriminatorios, trabaja para que sus instalaciones físicas sean accesibles, para que sus colaboradores y colaboradoras conozcan y practiquen el respeto a las diferencias; una empresa inclusiva garantiza que sus productos y servicios sean de acceso universal para todos y todas sus clientes y es consciente de que todas las personas son capaces de aportar valor a su organización.

Es importante destacar la Red de Empresas Inclusivas de Costa Rica que promueve el Plan Nacional de Inserción Laboral de PcD en Costa Rica, al desarrollar una iniciativa llamada Caja de Herramientas para Empresas Inclusivas, compuesta de nueve módulos que incluyen guías, manuales y matrices dirigidos a aquellas empresas que decidan ser inclusivas (Red de Empresas Inclusivas Costa Rica, 2013).

Los módulos de la Caja de Herramientas para Empresas Inclusivas de Costa Rica incluyen el autodiagnóstico empresarial y pasos para la elaboración de una política empresarial inclusiva de PCD; el análisis de puestos de trabajo y nivel de exigencia; los incentivos fiscales a las empresas; la inclusión de la discapacidad como estrategia de negocios; reclutamiento, selección e inducción; promoción de una cultura de inclusión en la empresa; el espacio físico y transporte; los ajustes razonables; y una guía de recursos y servicios para la inclusión.

Un módulo clave para cumplir con los demás es el que involucra la cultura, ya que para lograr inclusión óptima requiere un cambio de mentalidad y cultura reflejada en actitudes y promovida en todos los colaboradores. 
En el foro Perspectivas de la Situación Laboral de las PCD se manifestó: "Las barreras actitudinales constituyen la mayor limitante que enfrenta la población con discapacidad a la hora de insertarse en el mercado laboral" (Fonseca, 2013). Entonces, las empresas inclusivas promueven una cultura inclusiva, trabajan en accesibilidad y mejoran el entorno, incluyen políticas y procedimientos sobre el tema, involucran tecnología, adaptándose a formas de información y comunicación inclusivas, además de contar con servicios de apoyo y cumplir con la legislación nacional.

Estas empresas inclusivas se preocupan por eliminar barreras, como expone MeléndezLabrador (2016) pueden se barreras generales como las físicas, fisiológicas, semánticas y psicológicas o bien barreras de comunicación organizacional que pueden afectar la inclusión laboral de las PcD y su desenvolvimiento en el trabajo.

Por consiguiente, son necesarias empresas dispuestas a la inclusión, facilitando oportunidades de empleo valorando a las $\mathrm{PCD}$, ya que al final el trabajo también juega un papel central en la vida del ser humano, al que generalmente se le dedica muchas horas del día, el trabajar es dignificante y necesario para satisfacer diversas necesidades (Peressotti, 2017, p. 87).

Para finalizar está sección, aunque se habla más sobre el tema de inclusión y PcD con reconocimiento jurídico, aun hace falta mucho para reducir la brecha socioeconómica y aumentar la justicia para grupos que se encuentren en vulnerabilidad con acciones reales y como contribución a esta búsqueda interminable de igualdad siempre es positivo las investigaciones y diagnósticos que aportan al tema (Stang, 2011).

\section{Método de la investigación}

Se realizó una investigación no experimental transeccional descriptiva de las implicaciones en empresas y las PcD en relación con el tema de inclusión laboral. La investigación busca hacer aportes para la resolución de problemas en un conjunto de pasos o proceso aplicado a un fenómeno. Los estudios descriptivos son útiles para mostrar o describir con precisión las dimensiones de un fenómeno (Hernández, Fernández y Baptista, 2014).

Se aplicaron tres técnicas de investigación, la revisión literaria, encuesta y entrevistas cualitativas. La revisión literaria permite un marco referencia que orienta la discusión facilitando el planteamiento de las interrogantes en el trabajo de campo; por otra parte las entrevistas cualitativas permiten conocer experiencias, opiniones, valores, creencias, emociones, sentimientos e historias de vida (Hernández et al., 2014); y finalmente la encuesta permite valorar el punto de vista empresarial, identificando la perspectiva de los involucrados, sus conocimientos y percepciones sobre el tema, además describir hallazgos para comprender las implicaciones de la inclusión laboral para las empresas y las PcD.

La muestra estudiada en la encuesta fue de 381 empresas costarricenses, utilizando un muestreo probabilístico aleatorio simple con un nivel de confianza del 95\% y margen de error del 5\%. La población es finita y compuesta por empresas inscritas en el Directorio 
de Empresas y Establecimientos de Costa Rica, al momento de la investigación ascendían a 35.429 empresas (Instituto Nacional de Estadísticas y Censos de Costa Rica, 2019c). Los cuestionarios de la encuesta se aplicaron de dos maneras, en primer lugar por vía telefónica y posteriormente por medio de los formularios de Google en medio digital, además, sobre el tratamiento de los datos se les indicó a los participantes que la información aportada sería confidencial y usada únicamente para la presente investigación.

Con la intención de tener de primera mano experiencias y opiniones de las PcD, a través de entrevistas, como criterios de selección de estos casos de estudio, de manera intencional se buscaron PCD que se encontraran laborando y que al menos uno de los entrevistado tuviera un tipo de discapacidad distinta a los otros; es así como se establece la comunicación y se aplica la entrevista a una muestra intencional por conveniencia de tres personas con discapacidad: un hombre con limitación visual, una mujer también con limitación visual y una segunda mujer con limitación para caminar, o usuaria de silla de ruedas. Los tres entrevistados poseen grados universitarios, dos de ellos licenciatura y una con maestría profesional.

\section{Resultados ${ }^{2}$}

La información para analizar los resultados se obtiene de la encuesta y las entrevistas, como datos generales de los 381 encuestados representantes de empresas de Costa Rica, $63 \%$ son masculinos y $37 \%$ femeninos, con edades de 25 a 55 años. El $10 \%$ posee primaria completa, $37 \%$ secundaria completa y un $53 \%$ con grado universitario, cabe agregar que la mayoría de las empresas estudiadas fueron del sector comercio y servicios.

Considerando los objetivos de la investigación, se desarrollan en los siguientes puntos los resultados obtenidos con su discusión correspondiente. Inicialmente, destaca que la mayoría de encuestados no ha tenido la experiencia de laborar con una PcD, siendo un $92 \%$ los encuestados quienes afirmaron que en la empresa no se había contratado nunca un colaborador con discapacidad.

Del estudio, 343 empresas (90\%) consideran que el Gobierno, en los últimos cinco años, no ha promovido la inclusión laboral de las $\mathrm{PcD}$, este es un dato relevante considerando las iniciativas mencionadas en la revisión literaria, con avances jurídicos y las diversas entidades que se han involucrado en este campo, como el MTSS, MEP, CONAPDIS y otros buscando generar avances en la inclusión que según el estudio no se percibe en gran medida en el contexto empresarial.

En relación con los entrevistados coinciden con esta respuesta y al consultarles sobre los motivos indican que no se ha pasado del papel a las acciones. Al respecto uno de los entrevistados, Chaves (2018) afirmó:

2 El autor desea reconocer los aportes de Rita Corea Erazo, Carlos Chaves y Damaris Solano Murillo, PcD quienes compartieron sus conocimientos y experiencias sobre la necesidad de promover la inclusión laboral en las PcD de Costa Rica, esto es importante para el conocimiento de la situación actual y la generación de conciencia sobre este tema. 
Pueden hacerse leyes, pero no se ha dado el seguimiento adecuado, por ejemplo, establecer acciones específicas para que las instituciones contraten determinado número de PcD o que al menos sea evidente una apertura de plazas o puestos de trabajo inclusivos, además esas instituciones deben ir a las empresas y ver la realidad, buscar capacitarlos, no solamente hablar de lo que se quiere hacer sino hacerlo realmente.

La inclusión brinda beneficios a las empresas cómo reducciones en el pago de impuestos o premios, sobre el conocimiento de algunos de estos beneficios, sobresalió que el $49 \%$ no conoce ninguno y de los beneficios el más conocido es por gastos deducibles en impuesto sobre la renta que solo lo conocía el $14 \%$ de los encuestados, los otros beneficios consultados incluían Premio Costa Rica Incluye y la reserva de al menos $5 \%$ de las vacantes en los Poderes del Estado de Costa Rica para PcD.

Por su parte, los entrevistados conocen los beneficios expuestos e inclusive la Licda. Rita Corea Erazo agregó uno más a la lista: "El beneficio Mi Primer Empleo del MTSS paga aproximadamente 1.400 .000 colones en dos tractos, si se tiene en la empresa a la PCD al menos un año, esto para que la empresa lo utilice en las adaptaciones requeridas" (Corea, comunicación personal, 2018).

Considerando demostrar las implicaciones de la inclusión laboral de las PcD en las empresas, se destacó inicialmente la poca disposición de las empresas encuestadas para la inclusión de PcD, ya que solo 31\% estarían dispuestas a contratar una PcD.

Estas empresas contratarían personas con limitación para caminar, subir gradas o limitación para hablar. La limitación que sería menos aceptable en estas empresas es la limitación para utilizar brazos y manos, solamente $8 \%$ aceptaría está condición. Asimismo, se destaca que solo la mitad de las empresas dispuestas a contratar PcD, contrataría una persona con limitación para ver, este es un punto relevante ya que es la discapacidad más común.

Además, sobre los tipos de puestos dispuestos a contratar, las empresas con disposición contratarían para servicio al cliente y solo $29 \%$ afirmó que en puestos gerenciales. En los entrevistados, todos consideraron que una persona, sin importar la discapacidad, puede ser considerada en una empresa y, en cuanto a tipos de puesto, puede ser gerencial o cualquier otro, esto "dependerá más de los conocimientos, las capacidades y la actitud de la persona" (Corea, comunicación personal, 2018).

Por otra parte, en la figura 3 se presentan los retos consultados que puede enfrentar una PcD para conseguir empleo, entre estos destacó que $79 \%$ de las empresas encuestadas considera el reto de la falta de perfiles de puesto con las condiciones inclusivas para que una PCD pueda ejecutarlo, siendo este el principal reto arrojado en el estudio.

Así también, 56\% consideró la inversión en adaptaciones físicas como reto de las empresas para la inclusión laboral. El $44 \%$ considera relevante los cambios en procesos 
de reclutamiento y selección, así como también destacan barreras actitudinales y necesidad de capacitaciones al personal sobre inclusión.

Fal ta de perfiles de puesto adecuados

Inversión en adaptaciones físicas

Cambios en procesos de reclutamiento y selección

Barreras actitudinales por prejuicios

Fal ta de capacitación en el personal de la empresa

Barreras arquitectónic as y físicas para las PcD

Fal ta de acceso a tecnología de apoyo

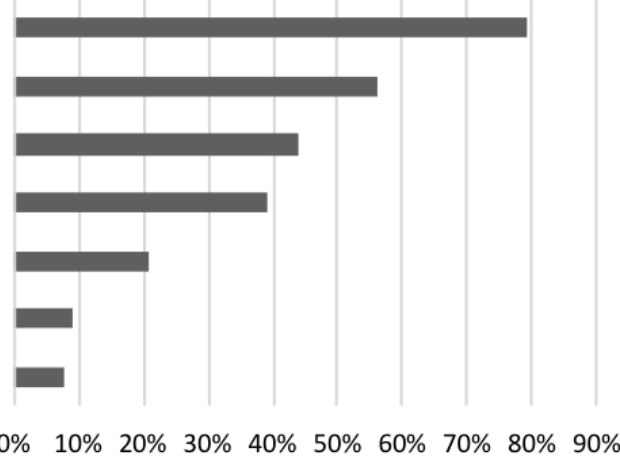

Figura 3. Empresas costarricenses encuestadas (retos para la inclusión laboral): percepción de retos para la inclusión laboral de PcD (en porcentajes).

Fuente: Elaboración propia sobre la base de encuesta aplicada a 381 empresas costarricenses para conocer la percepción sobre los retos que debe enfrentar una persona con discapacidad para ser incluida en el ámbito laboral, 2019.

Las personas entrevistadas coinciden en la importancia de las capacitaciones para el personal en temas de inclusión y los cambios en procesos de reclutamiento y selección ya que estos también aportarían a generación de los puestos adecuados. Además, indican que el reto más importante es actitudinal; además, uno de los entrevistados agregó otro reto adicional: "el transporte y acceso externo, como usuaria de silla de ruedas me influye la condición de las calles con huecos, aceras sin rampas y entradas con gradas, falta semáforos, etc." (Solano, comunicación personal, 2018).

Por su parte, Carlos Chaves (comunicación personal, 2018) indicó: "Algunos retos van a depender de cada tipo de discapacidad y del nivel de afectación de esa condición, por ejemplo, para el no vidente la tecnología es clave, como software lector o lupa electrónica, mientras que para un usuario de silla de ruedas la barrera arquitectónica adquiere importancia".

Cabe agregar que $65 \%$ de las empresas encuestadas consideran positiva la percepción de los clientes sobre la empresa al notar que dentro de la organización se incluye una PCD. Considerando realizar recomendaciones que permitan mayor inclusión laboral de PcD en las empresas, el $49 \%$ indicó que se logra con más capacitaciones a empresas sobre este tema, un $27 \%$ indicó que con más publicidad a nivel país, y $24 \%$ con más beneficios legales para las empresas.

Sobre este punto, los entrevistados coinciden en las capacitaciones; además, Chaves agrega "considerar la capacitación vivencial como talleres, y que sea una PcD que lo 
exponga ya que es él o ella la que sabe lo que se requiere por las propias experiencias" (Chaves, comunicación personal, 2018).

Por otra parte, buscando medidas que permitan una mayor inclusión laboral de las PcD en las empresas, la mayoría de encuestados consideran, que lo más importante es el apoyo de las entidades públicas según afirmó el $41 \%$ de los encuestados, además $30 \%$ consideró las bolsas de empleo inclusivas y $29 \%$ destacó la relevancia de promover la educación inclusiva.

Considerando las entrevistas, opinan que el apoyo de las entidades públicas es lo más importante y promover más la educación inclusiva. Una de las entrevistadas comentó que la educación es una clave fundamental, ya que esto puede ir aportando una cultura inclusiva, "si a los niños desde la escuela les enseñan sobre la igualdad de oportunidades y no excluir a otras personas por tener alguna situación de discapacidad, tendríamos más esperanza para las generaciones futuras en la inclusión" (Corea, comunicación personal, 2018).

Las recomendaciones dadas por encuestados a las empresas para la inclusión incluyen: no discriminar, brindar oportunidades, disposición para aprender, tener mente abierta a cambios, innovar en perfiles de puestos, evaluar perfiles donde las limitaciones no sean impedimento, que se informen y capaciten sobre el tema y dar igualdad de oportunidades.

Además, sobre las recomendaciones de encuestados para que las PcD tengan más oportunidades de empleo, destacan que se capaciten, sean autocríticos manteniendo alta autoestima ya que todos los seres humanos son valiosos, estudien áreas y busquen empleo donde puedan tener un buen desempeño.

Considerando los aportes dados por las PcD entrevistadas para mayor inclusión laboral, las recomendaciones para las empresas se centran en capacitación en todos los niveles principalmente en los niveles gerenciales donde se toman decisiones de contratación, también se necesitan cambios en las características de ofertas laborales ajustando los perfiles de puestos, haciéndolos perfiles inclusivos.

Más aún, lograr incentivar una visión de las adaptaciones como una inversión y no como gasto más, y esto se puede lograr promoviendo la participación y escuchando a las PcD que son las que de primera mano saben cuáles son las necesidades de adaptación.

En los procesos de gestión del talento humano se comentó el tema de mejorar el acceso a la información en los procesos empresariales para que sean más inclusivos, como ejemplo el uso de Braille. También se invita a que las empresas pierdan miedo, se animen y se capaciten, ya que con la inclusión se pueden obtener beneficios de imagen con los colaboradores, aporta motivación y promueve valores. También aprovechar tecnologías de información y comunicación, aplicar iniciativas como teletrabajo por la movilidad que puede generar una barrera en las $\mathrm{PcD}$ e investigar empresas que tienen experiencia con PcD y aprender de estas. Además, informarse sobre los avances tecnológicos aplicables, 
que también constantemente tienen mejoras que pueden ser útiles tanto para las PcD como para las empresas fomentando así la inclusión.

Como recomendaciones para las $\mathrm{PcD}$, los entrevistados aportaron que es positivo buscar capacitaciones, por ejemplo, computación y segundo idioma; además, de ser posible, a nivel universitario, ya que como afirmó Chaves (comunicación personal, 2018) "los títulos universitarios hacen ver en el empleador la actitud y el esfuerzo hecho y esto aumenta en gran manera las posibilidades de contratación". También recomendaron invertir tiempo en habilidades blandas, como trabajo en equipo, liderazgo, comunicación, relaciones e inteligencia emocional, ya que permitirá llevar a cabo de mejor manera tanto las actividades cotidianas como las de un empleo.

Mencionan, además, la relevancia de que la PcD proyecte seguridad, autoestima, empoderamiento, que conozca legislación, que se valore, desarrolle autonomía, hacer un buen currículo e investigar sobre la empresa donde quiere laborar, que no quiera ser visto como un recurso humano sustituible, sino protagonista, capaz de grandes cosas y también se comentó la importancia de cuidar la presentación personal (Corea, comunicación personal, 2018).

Para finalizar, acerca de lo que hace falta a nivel país, en la entrevista indicaron la importancia sobre el cumplimiento eficaz de legislación, por ejemplo, sanciones por incumplimiento de las empresas. Además, considerando que son siglos de exclusión y del "pobrecito", se deben eliminar barreras y, como principal, la actitudinal, hay muchas personas que no han compartido con una PcD, eso disminuye la sensibilidad, "si no se tiene la experiencia hay que crear conciencia, es incluso un tema cultural que a su vez se trasmite a las organizaciones (Chaves, comunicación personal, 2018).

\section{Conclusiones}

La inclusión laboral de las PCD es un tema importante para Costa Rica generando leyes específicas como las mencionadas en la revisión literaria, pero las acciones y los resultados no están cumpliendo las expectativas de acuerdo con la investigación. Considerando los objetivos planteados al inicio del artículo se determinan las siguientes conclusiones.

Hay desconocimiento en las empresas sobre los beneficios de contratar PcD, no se percibe que el Gobierno y entidades públicas promuevan la inclusión por falta de acciones tangibles y para las empresas es relevante el cumplimiento de los perfiles de puesto y la inversión en adaptaciones físicas.

Por su parte, las PcD afirman la importancia de capacitaciones en inclusión, cambios en procesos de reclutamiento y selección, así como tratar de informar para combatir las barreras actitudinales. Considerando estas implicaciones se recomienda que las empresas se informen, capaciten y promuevan una cultura organizacional inclusiva, con visión estratégica, considerando oportunidades, desde la percepción positiva de clientes hasta beneficios económicos en impuestos, premios o remuneraciones. 
Con relación a los procesos de gestión del talento humano en las empresas, es importante que sean inclusivos en ofertas laborales y en igualdad de oportunidades, por ejemplo, al momento de hacer pruebas o entrevistas considerar adaptaciones, así como generar perfiles de puestos inclusivos facilitando la igualdad de oportunidades. También valorando la generación de espacios inclusivos con mayores oportunidades y por lo tanto aportar a una mejor calidad de vida en PcD.

Las empresas pueden aprender de las PcD, Chaves (2018) explicó que se debe tener una visión de discapacidad y aprovechar información de la misma persona que tiene una discapacidad, muchas veces las personas cometen errores y hacen exclusión por desconocimiento, la mejor forma para aprender sobre inclusión es escuchando y aprendiendo de las mismas PCD, "para las empresas implica hasta menores costos, llevar una PcD para que asesore o recomiende adaptaciones, a veces se gasta dinero con profesionales que no conocen la realidad de la discapacidad y al final las cosas no cumplen su función inclusiva, mejor primero escuche a la PcD" (Chaves, comunicación personal, 2018).

La percepción de que la inclusión es cara y que requiere muchos cambios, se puede reducir informándose y aprendiendo que las adaptaciones pueden ser tan sencillas como quitar una esquina a una tarjeta para que un no vidente pueda reconocerla (Solano, 2018).

La desocupación en PcD es afectada por la poca disposición para contratar. Además, las implicaciones para que contraten van a depender de la discapacidad y el grado de esta, por ejemplo, según la presente investigación, personas con limitación para caminar tienen más posibilidades que un no vidente, esto es considerable ya que alrededor del $50 \%$ de las PcD del país tiene limitación visual. También, las empresas consideran como retos principales la falta de perfiles de puestos inclusivos y las inversiones en adaptaciones, mientras que la PcD considera que son más las barreras actitudinales.

Entonces, es recomendable considerar la importancia de la capacitación y el desarrollo profesional de las PcD; además, la información en materia legal y asesoría de entidades como Conapdis y en relación con el tema actitudinal se recomienda la interacción con otras PcD donde se puedan obtener consejos sobre experiencias.

Por su parte, la PcD debe demostrar autonomía, aprender constantemente y adaptarse, por ejemplo, una persona con limitación visual debería conocer y esforzarse por buscar, obtener y usar herramientas tecnológicas. Así también, cabe agregar, como indica Canossa (2019) "se ha incrementado la competitividad en las empresas, de ahí que se exijan más requisitos a la hora de contratar talento humano, ya que no se necesita que las personas posean solo el conocimiento, sino también que tengan desarrolladas las habilidades blandas", este es un buen tema para investigaciones futuras donde se estudien las habilidades blandas y la propuesta de acciones concretas para promoverlas en PcD.

Las empresas consideran que la capacitación es clave para la inclusión, así como el apoyo de otras entidades para un mayor acceso a empleo. Además, es necesario en las empresas la apertura a PCD y adaptarse considerando beneficios. Claramente las PCD 
deben capacitarse para cumplir con las aptitudes, competencias y habilidades requeridas y ser consideradas basándose en las capacidades y no las limitaciones.

Por consiguiente, se requiere una mayor comunicación sobre los beneficios, leyes e iniciativas sobre inclusión de las PcD, mediante publicidad en medios; además, en agrupaciones como cámaras de sectores empresariales, ya que si están más informadas las posibilidades de inclusión podrían aumentar.

Además, el Gobierno, mediante entidades públicas, debe accionar sobre capacitaciones, tanto para empresas como para $\mathrm{PcD}$, incrementando oportunidades laborales, por ejemplo, con Empléate Inclusivo. La MSc. Damaris Solano, consultora en discapacidad y entrevistada, afirma: "No solo es capacitar en áreas de demanda laboral, sino que se dé seguimiento sobre la situación laboral de la persona capacitada, de esta forma se puede mejorar, no solo con capacitación sino con colocación" (Solano, 2018).

Finalmente, la inclusión laboral de PcD es un tema no solo de derechos sino también de ética y acciones concretas, queda mucho por mejorar, se deben continuar haciendo estudios, generando estadísticas y describiendo el fenómeno de inclusión, mostrando el problema y la realidad del mismo para crear conciencia, e indudablemente el principal cambio empieza por nosotros aportando para ser una sociedad más inclusiva y respetuosa, promoviendo la calidad de vida y, así, el desarrollo de las empresas y los países.

\section{Referencias}

Barquero, K. (8 de abril, 2016). Más de 350 PcD participaron en Empléate. $C R$ Hoy. Recuperado de http://www.crhoy.com/archivo/ mas-de-350-personas-con-discapacidad-participaron-en-empleate/nacionales/

Canossa, H. (2019). Habilidades blandas en el estudiantado universitario y la empleabilidad en Costa Rica. Revista Académica Arjé. 2(2), 5-13. Recuperado de https://revistas.utn.ac.cr/ index.php/arje/article/view/205

Chaves, C. (10 de junio, 2018). Comunicación personal. (entrevista sobre inclusión laboral). Liberia, Costa Rica.

Corea, R. (9 de agosto, 2018). Comunicación personal. (entrevista sobre inclusión laboral). Liberia, Costa Rica.

Fonseca, M. (1 de julio, 2013). Barreras actitudinales limitan inserción laboral de población con discapacidad. Universidad de Costa Rica. Recuperado de https://www.ucr.ac.cr/noticias/2013/07/01/barreras-actitudinales-limitan-insercion-laboral-de-poblacion-con-discapacidad.html

Gonzáles, D. y Stang, F. (2014). Las PcD en América Latina a 20 años de los consensos de El Cairo: la necesidad de información para políticas y programas. Notas de Población, CEPAL, 99(1), 67-106. Recuperado de https://doi.org/10.18356/6856cafo-es

Hernández, R.; Fernández, C. y Baptista, M. (2014). Metodología de la Investigación (Sexta Ed.). México: McGraw Hill.

Instituto Nacional de Estadísticas y Censos. (INEC). (2019a). Enadis 2018. Población por situación de discapacidad, según condición de actividad y características del empleo. INEC. Recuperado de http://www.inec.go.cr/social/poblacion-con-discapacidad 
Instituto Nacional de Estadísticas y Censos (2019b). Enadis 2018. Población por situación de discapacidad, según nivel de instrucción y características generales y de accesibilidad. INEC. Recuperado de http://www.inec.go.cr/social/poblacion-con-discapacidad

Instituto Nacional de Estadísticas y Censos de Costa Rica (2019c). DEE.2018. Total de empresas según sectores de actividad económica a un dígito por intervalo de trabajadores. INEC. Recuperado de http://www.inec.go.cr/economia/directorio-de-empresas-y-establecimientos-0

Instituto Nacional de Estadísticas y Censos de Costa Rica (2011). Censo. 2011. Población total por condición y tipo de discapacidad, según provincia, sexo y grupos de edad. INEC. Recuperado de https://www.inec.cr/censos/censos-2011

Instituto Nacional de Estadísticas y Censos y Consejo Nacional de Personas con Discapacidad de Costa Rica (2019). Encuesta Nacional sobre Discapacidad 2018. Resultados Generales. Área de Censos y Encuestas. Recuperado de http://www.inec.go.cr/sites/default/files/documetos-biblioteca-virtual/reenadis2018.pdf

Ley N 7092 (1988). Ley del Impuesto sobre la Renta. Publicado en el Diario Oficial La Gaceta el 19 de mayo de 1988.

Ley N 7600 (1996). Igualdad de Oportunidades para las PcD. Publicado en el Diario Oficial La Gaceta No. 112, del 29 de mayo de 1996.

Ley № 8661 (2008). Aprobación de la Convención sobre los Derechos de las Personas con Discapacidad. Decreto Ejecutivo $\mathrm{N}^{\circ} 34780$ de 29 de setiembre de 2008. Presidencia de la República. San José, 19 de agosto del 2008.

Ley ํㅛ 8862 (2010). Ley de Inclusión y Protección Laboral de las PcD en el Sector Público. Publicada en La Gaceta n. 219 de 11 de noviembre de 2010.

Ley N 9303 (2015). Ley de creación del Consejo Nacional de PcD. Publicada en La Gaceta no 123 de 26 de junio del 2015.

Mathis, R. L.; Jackson, J. H. y Valentine, S. R. (2014). Human Resource Management. (14 Ed.). Connecticut, USA: Cengage Learning

Meléndez-Labrador, S. (2016). Comunicación interna incluyente. Dos estudios de caso de inclusión laboral de personas con discapacidad auditiva en Bogotá. Investigación \& Desarrollo, 24(1), 26-52. DOI: http://dx.doi.org/10.14482/indes.24.1.8683

Mercado, E.; Aizpurúa, E. y García, L. M. (2013). Avanzando hacia la igualdad de oportunidades en la inclusión socio-laboral de las PcD. Cuadernos de Trabajo Social, 26(1), 95-104. https:// doi.org/10.5209/rev_CUTS.2013.v26.n1.39571

Millán, P.; Arredondo F. G. y Vázquez J.C. (2018). Inclusión laboral, paz y sostenibilidad. Methaodos. Revista de Ciencias Sociales, 6(2) 174-189. https://doi.org/10.17502/m.rcs.v6i2.233

Ministerio de Educación Pública de Costa Rica. (MEP). (2019). Centro de Educación Especial. Gobierno de Costa Rica. Recuperado de https://www.mep.go.cr/programas-y-proyectos/ yo-me-apunto/centros-educacion-especial

Ministerio de Trabajo y Seguridad Social de Costa Rica. (2012). Plan Nacional de inserción laboral para la población con discapacidad en Costa Rica. Recuperado de http://www.mtss.go.cr/ seguridad-social/discapacidad.html

Ministerio de Trabajo y Seguridad Social de Costa Rica (2019). Departamento de Igualdad de Oportunidades para las Personas con Discapacidad. MTSS. Recuperado de http://www.mtss. go.cr/seguridad-social/discapacidad.html

Organización Internacional del Trabajo. (2015). Estrategia y plan de acción para la inclusión de la discapacidad 2014-2017. Recuperado de http://www.ilo.org/wcmsp5/groups/public/--ed_emp/---ifp_skills/documents/genericdocument/wcms_370773.pdf 
Organización de las Naciones Unidas. (ONU) (10 de diciembre, 1948). Declaración Universal de Derechos Humanos. Recuperado de http://www.ohchr.org/EN/UDHR/Documents/UDHR_ Translations/spn.pdf

Peressotti, C. (2017). Inclusión laboral de PCD: metodología y práctica. Córdoba: Editorial Brujas

Presidencia de la República de Costa Rica. (23 de enero, 2017a). MTSS realizará talleres para impulsar la inclusión laboral de las PcD en el sector público. Recuperado de http://presidencia.go.cr/comunicados/2017/01/mtss-realizara-talleres-para-impulsar-la-inclusion-laboral-de-las-personas-con-discapacidad-en-el-sector-publico/

Presidencia de la República de Costa Rica. (12 de enero, 2017b). 9 empresas son reconocidas por sus buenas prácticas de inclusión social y laboral de PCD. Recuperado de http://presidencia.go.cr/comunicados/2017/01/9-empresas-son-reconocidas-por-sus-buenas-practicas-de-inclusion-social-y-laboral-de-personas-con-discapacidad/

Red de Empresas Inclusivas Costa Rica. (2013). Aumente la competitividad de su empresa. Recuperado de http://www.aedcr.com/redempresasinclusivas/

Solano, D. (15 de julio, 2018). Comunicación personal (entrevista sobre inclusión laboral). Liberia, Costa Rica.

Stang, M. F. (2011). Las PcD en América Latina: del reconocimiento jurídico a la desigualdad real. Serie Población y Desarrollo. Santiago: CELADE. Recuperado de https://repositorio.cepal. org/bitstream/handle/11362/7135/1/S1100074_es.pdf

Victoria, J. A. (2013). El modelo social de la discapacidad: una cuestión de derechos humanos. Revista de Derecho UNED, 12(1), 817-833. https://doi.org/10.5944/rduned.12.2013.11716 